the initial 52 subjects enrolled. Participants were assessed once over 24 hours by ambulatory blood pressure monitoring, using routine equipment and SpaceLabs software. Diurnal and nocturnal averages for both systolic and diastolic blood pressure were assessed, and nocturnal blood pressure dipping was calculated as difference between median awake and sleep measurements. In addition, blood pressure loads were calculated as percentages of measurements that exceeded validated, age-specific thresholds for normal blood pressure. Nocturnal hypertension was defined as systolic or diastolic load during sleep of $>25 \%$. Enrollment in the BPpSLE study is open to all interested CARRA investigators.

Results The cohort is 79\% female, 29\% Hispanic and 27\% black. Median age was 16.3 years (IQR 13-17) at time of testing. Median SLEDAI was 4 (IQR 2-11), renal SLEDAI was 0 (IQR 0-4), and median glomerular filtration rate was $104 \mathrm{~mL} / 1.73 \mathrm{~m}^{2} / \mathrm{min}$ (IQR 91-114). Nocturnal hypertension was seen in $47 \%$ and attenuated dipping was seen in $71 \%$ of children with SLE, compared to $39 \%$ and $26 \%$ of historical controls. Loads were $14 \%$ and $13 \%$ while sleeping verses 5\% systolic and $6 \%$ diastolic while awake. This corresponded with median nocturnal dipping of $6 \%$ in systolic and $13 \%$ in diastolic blood pressures (normal $>10 \%$ ).

Conclusions There is a high prevalence of both nocturnal hypertension and attenuated nocturnal blood pressure dipping in out SLE cohort, compared to an age-matched adolescent population with borderline high blood pressure. These patterns are not due to renal failure or active nephritis. Although most SLE patients in the cohort do not meet criteria for diagnosis of hypertension, the degree of dysregulation could potentially contribute to the cardiovascular risk noted in SLE.
Acknowledgements This abstract is being presented on behalf of the Midwest Pediatric Nephrology Consortium (MWPNC).

\section{CS-35 DAMAGE ACCRUAL AND HEALTH-RELATED QUALITY OF LIFE IN A COLOMBIAN COHORT OF PATIENTS WITH SYSTEMIC LUPUS ERYTHEMATOSUS}

1,2Sebastian Herrera Uribe*, 'Marcela Posada Velasquez, ${ }^{1,2}$ Juan Camilo Diaz-Coronado, ${ }^{1,2,3}$ Adriana Rojas-Villarraga, 'Deicy Hernández-Parra, ${ }^{1}$ Paula Perez-Estrada, ${ }^{1}$ Ricardo Pineda-Tamayo. ${ }^{1}$ Clinical information group, Artmedica IPS, Medellin, Colombia; IInternal Medicine Department, CES University, Medellin, Colombia; ${ }^{3}$ División Investigaciones, Fundación Universitaria Ciencias de la Salud FUCS, Bogotá, Colombia

\subsection{6/lupus-2018-Ism.70}

Background Systemic Lupus Erythematosus (SLE) is a chronic multisystemic autoimmune pathology of unknown etiology with a variable course and a predilection for women of reproductive age. Chronic activity and flares of disease activity are correlated with greater damage accrual and mortality. Mortality and health-related quality of life (HR-QoL) are higher in SLE patients when compared to general population. Damage accrual, duration of the disease, disease activity and involvement of specific organ systems, might relate to worse HRQoL in SLE. A worse perception of health is associated with a higher damage accrual over time.

Methods A retrospective cohort of SLE patients (ACR 1997 or SLICC 2012 classification criteria), followed for at least 1 year, between 2015 and 2017 in a specialized center was analyzed. Activity evaluated by Systemic Lupus Erythematosus Disease Activity Index (SLEDAI), damage accrual by SLICC/

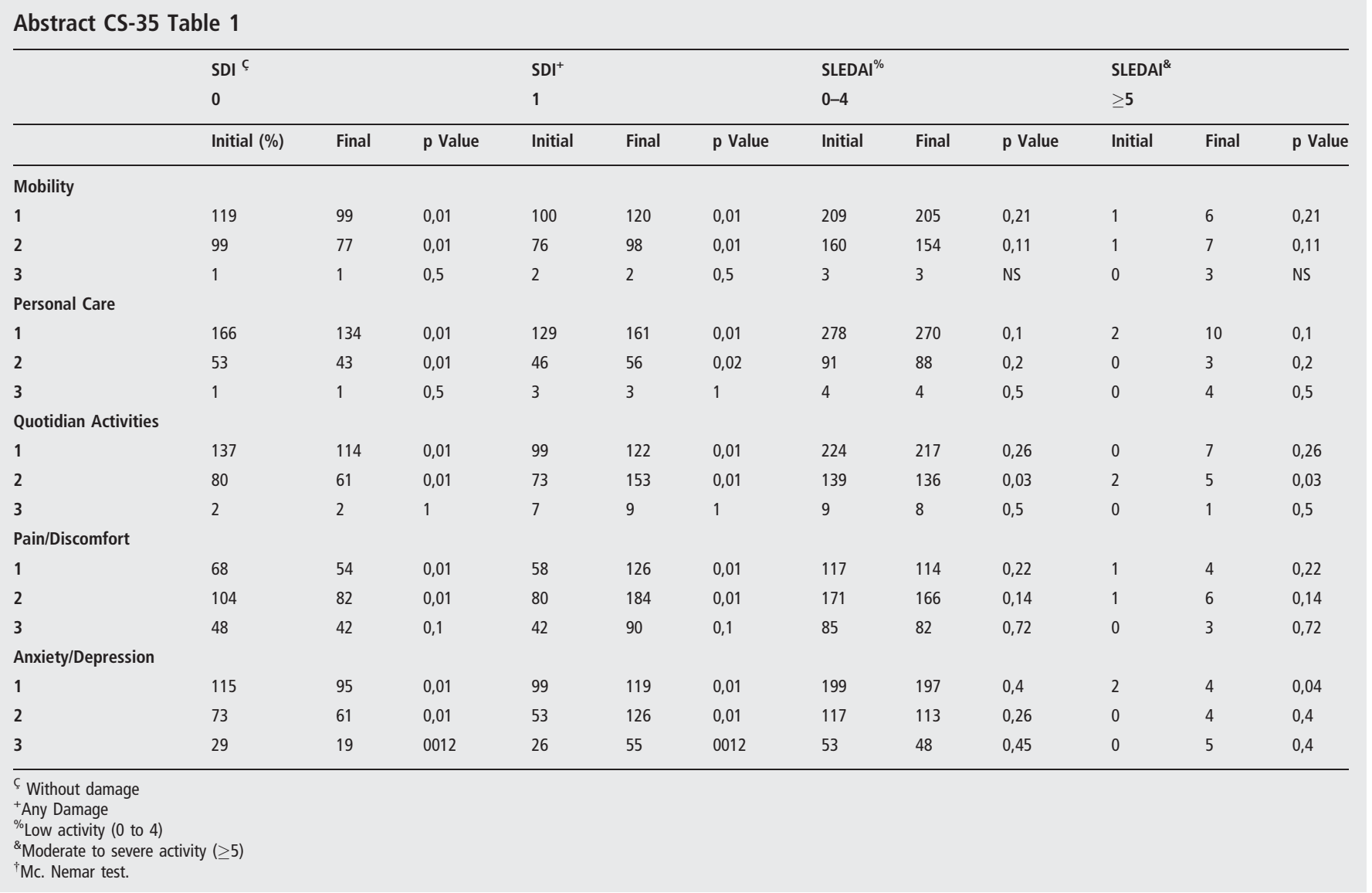


ACR damage index (SDI) and HR-QoL by EQ-5D were measured. Bivariate analysis through chi squared and U Mann Whitney Multivariate analysis was performed by logistic regression to adjust for significant associations. Statistical analysis for related samples was evaluated with Mc. Nemar test.

Results We analyzed 400 Colombian patients. Baseline median age was 49 years (15 IQR) with median age at diagnosis and disease duration of 37 years (17 IQR) and 9 years (13 IQR) respectively. There were $94 \%$ female patients and $17.3 \%$ late onset SLE. Most frequent clinical manifestations were hematological (82.8\%), mucocutaneous (75.3\%) and nephritis (33.8\%). Only $4.5 \%$ had neurological involvement. The mean SLEDAI were 1.18 and 0.65 at first and second measurement respectively, in the first measurement $97.1 \%$ of the patients had a SLEDAI $\leq 4$. The mean SDI was 0.7275 at first measurement and 0.985 at the second measurement. Although SDI was associated to various dimensions of HR-QoL measured by ED-5D, disease activity was not related (See table 1).

Conclusions In SLE Colombian patients with a stablished disease and an altered HR-QoL, low disease activity is not related with HR-QoL when measured by EQ-5d. In the present study, it is highlighted that while disease activity decreases, damage increases. Damage accrual has a relation with HR-QoL in the short term. The impact and correlation must be better defined in a long-term follow-up. The associated effect on HRQOL emphasizes the need for strategies to reduce the risk of cumulative organ damage.

\section{CS-36 RECOMMENDATIONS FOR THE ASSESSMENT OF SYSTEMIC LUPUS ERYTHEMATOSUS IN CANADA}

${ }^{1}$ Stephanie Keeling*, ${ }^{2}$ Zainab Alabdurubalnabi, ${ }^{2}$ Antonio Avina-Zubieta, ${ }^{3}$ Susan Barr, ${ }^{4}$ Louise Bergeron, ${ }^{5}$ Sasha Bernatsky, ${ }^{6}$ Josiane Bourre-Tessier, ${ }^{3}$ Ann Clarke, ${ }^{6}$ Alexandra BarilDionne, ${ }^{2}$ Jan Dutz, ${ }^{2}$ Stephanie Ensworth, ${ }^{3}$ Aurore Fifi-Mah, ${ }^{7}$ Paul R Fortin, ${ }^{8}$ Dafna Gladman, ${ }^{9}$ Derek Haaland, ${ }^{10}$ John G Hanly, ${ }^{11}$ Linda T Hiraki, ${ }^{6}$ Sara Hussein, ${ }^{9}$ Kimberly Legault, ${ }^{11}$ Deborah Levy, ${ }^{12}$ Lily Lim, ${ }^{9}$ Mark Matsos, ${ }^{13}$ Emily G McDonald, ${ }^{8}$ Jorge Medina-Rosas, ${ }^{14}$ Jordi Pardo Pardi, ${ }^{12}$ Christine Peschken, ${ }^{5}$ Christian Pineau, ${ }^{15}$ Janet Pope, ${ }^{16}$ Tamara Rader, ${ }^{2}$ Jen Reynolds, ${ }^{11}$ Earl Silverman, ${ }^{8}$ Konstantinos Tselios, ${ }^{6}$ Manon Suitner, ${ }^{8}$ Murray Urowitz, ${ }^{8}$ Zahi Touma, ${ }^{5}$ Evelyne Vinet, ${ }^{17}$ Nancy Santesso. ${ }^{1}$ Divison of Rheumatology, Department of Medicine, University of Alberta; ${ }^{2}$ Division of Rheumatology, Department of Medicine, University of British Columbia; ${ }^{3}$ Division of Rheumatology, Department of Medicine, Cumming School of Medicine, University of Calgary; ${ }^{4}$ Louise Bergeron, Canadian Arthritis Patient Alliance; ${ }^{5}$ Division of Rheumatology, Department of Medicine, McGill University; ${ }^{6}$ Division of Rheumatology, Department of Medicine, Université de Montreal; ${ }^{7}$ Division of Rheumatology, Department of Medicine, Université Laval; ${ }^{8}$ Institute of Health Policy, Management and Evaluation, University of Toronto; ${ }^{9}$ Division of Rheumatology, Department of Medicine, McMaster University; ${ }^{10}$ Division of Rheumatology, Department of Medicine, Dalhousie University; ${ }^{11}$ Division of Rheumatology, Hospital for Sick Children, Department of Pediatrics University of Toronto; ${ }^{12}$ Division of Rheumatology, Department of Medicine, University of Manitoba; ${ }^{13}$ General Internal Medicine, McGill University Health Centre, McGill University; ${ }^{14}$ Cochrane Musculoskeletal Group, University of Ottawa; ${ }^{15}$ Division of Rheumatology, Department of Medicine, Western University; ${ }^{16}$ Canadian Agency for Drugs and Technologies in Health; ${ }^{17}$ Department of Health Research Methods, Evidence and Impact, McMaster University

\subsection{6/lupus-2018-Ism.71}

Background To develop GRADE-based recommendations for the diagnosis and monitoring of systemic lupus erythematosus patients in Canada.

Methods Recommendations were developed using the GRADE (Grading of Recommendations, Assessment, Development and Evaluation) approach. The Canadian SLE Working Group (panel of Canadian rheumatologists and patient representative
Abstract CS-36 Table 1 Summary of recommendations with strength of evidence for the diagnosis and monitoring of SLE in Canada

Recommendation

Strength of

Recommendation; Quality of

Evidence

We recommend that all adult and pediatric patients

suspected of SLE be referred to a lupus specialist, most strong recommendation;

often a rheumatologist, to confirm diagnosis and be involved in ongoing care

For adult and pediatric patients with SLE, we suggest assessing disease activity with a validated instrument of disease activity during baseline and follow up visits. For adult and pediatric patients with SLE, we suggest assessing disease activity with a validated instrument of disease activity during baseline and follow up visits For adult lupus patients, we recommend that indicators of obesity, smoking status, diabetes, blood pressure and a basic lipid profile be measured upon diagnosis of SLE and be reassessed periodically according to current recommendations in the general population and be used to inform the cardiovascular risk assessment.

For adult patients with SLE, we suggest assessing the risk of osteoporosis and fractures every 1 to 3 years using a detailed history and focused physical examination, and measuring bone mineral density in patients with other risk factors according to recommendations in the general population. For all adults with SLE, we suggest screening 25hydroxyvitamin $D$ as part of the assessment for risk of osteoporosis and fractures

For adults and paediatric patients with a diagnosis of SLE and high risk behaviours for HCV acquisition, we recommend screening for Hepatitis $\mathrm{C}$ and repeating according to recommendations in the general population. For all other adult and paediatric patients with a diagnosis of SLE we suggest screening for Hepatitis $C$ and repeating according to recommendations in the general population

For adult patients with SLE who do not have clinical symptoms suggestive of osteonecrosis, we suggest not screening for or performing investigations for osteonecrosis. For patients who have suspected clinical symptoms of osteonecrosis, we suggest radiographs as the initial imaging modality rather than MRI or bone scan with SPECT according to recommendations in the general population.

For women with SLE, we suggest that anti-Ro and antiLa antibodies be measured immediately prior to pregnancy or during the first trimester.

For pregnant women with SLE, we suggest that uterine and umbilical Doppler studies be performed in the second or third trimester, or at the time of a suspected flare.

For women with prior or active lupus nephritis who are pregnant, we suggest measuring serum creatinine and urine protein to creatinine ratio every 4-6 weeks, or more frequently if clinically indicated. We suggest blood pressure and urinalysis be measured prior to pregnancy and every 4-6 weeks until 28 weeks, every 1-2 weeks until 36 weeks and then weekly until delivery. moderate quality evidence

conditional recommendation, low quality evidence

conditional recommendation, low quality evidence strong recommendation, moderate quality evidence conditional recommendation, low quality evidence moderate quality evidence conditional recommendation, low quality evidence

conditional recommendation, low quality evidence

conditional recommendation, low quality evidence

conditional recommendation; low quality evidence

conditional recommendation; low quality evidence strong recommendation, 